\title{
Unpacking the Infrastructuring Work of Patients and Caregivers around the World
}

\author{
Yunan Chen \\ University of California, Irvine \\ Irvine, CA, USA \\ yunanc@ics.uci.edu
}

\section{Xinning Gui}

University of California, Irvine

Irvine, CA, USA

guix@uci.edu

\section{Claus Bossen \\ Aarhus University \\ Aarhus, Demark}

clausbossen@cc.au.dk

\section{Valeria Herskovic}

Pontificia Universidad Católica de Chile

Santiago, Chile

vherskov@ing.puc.cl

\author{
Nervo Verdezoto \\ University of Leicester \\ Leicester, UK \\ nervo.verdezoto@le.ac.uk
}

\section{Xiaojuan Ma}

Hong Kong University of Science and

Technology

Hong Kong SAR, China

mxj@cse.ust.hk

\section{Naveen Bagalkot}

Srishti Institute of Art, Design, and

Technology

Bangalore, India

naveen@srishti.ac.in

\section{Bernd Ploderer}

Queensland University of Technology

Brisbane, Australia

b.ploderer@qut.edu.au 


\section{ABSTRACT}

Many healthcare systems around the world are noted as fragmented, complex and low-quality, leaving patients and caregivers with no choice but to engage in "infrastructuring work" to make it function for them. However, the work patients do to construct their functioning health service systems often remain invisible, with very little resources and technologies available to assist them. This workshop aims to bring together researchers, health practitioners, and patients to examine, discuss, and brainstorm ways to re-envision our healthcare service systems from the perspective of patients and caregivers. We aim to unpack the types of work that patients and caregivers do to reconfigure, reconstruct and adapt the healthcare infrastructure, and to brainstorm design solutions that can provide better infrastructuring assistance to them.

\section{CCS CONCEPTS}

- Human-centered computing $\rightarrow$ Human computer interaction (HCI); User studies; • Applied computing $\rightarrow$ Consumer health;

\section{KEYWORDS}

Health Services; Healthcare Infrastructure; Patients' Work; Invisible Work; Caregivers Work; Engagement

\section{ACM Reference Format:}

Yunan Chen, Nervo Verdezoto, Xinning Gui, Xiaojuan Ma, Claus Bossen, Naveen Bagalkot, Valeria Herskovic, and Bernd Ploderer. 2019. Unpacking the Infrastructuring Work of Patients and Caregivers around the World. In Proceedings of CHI'19 Extended Abstracts. ACM, New York, NY, USA, ?? pages. https://doi.org/10.1145/3290607. $\mathrm{XXXXXXX}$

\section{INTRODUCTION}

While it is essential for individuals to obtain timely, affordable, and satisfactory healthcare services, they encounter numerous challenges interacting with the fragmented and often imperfect healthcare infrastructures. The challenges they face include but are not limited to: deciding whom and where to seek for medical service among numerous qualified medical doctors, puzzling out insurance and payment options, coordinating and communicating with multiple healthcare providers, selecting the optimal treatment plans, making sense of a vast amount of health data coming from different healthcare professionals, and negotiating socio-cultural boundaries between care settings, etc. These challenges force patients and caregivers to engage in ongoing "work" to (re)-configure, connect, communicate, construct, adapt and sustain different infrastructural arrangements between human and nonhuman entities (e.g., people, places, routines, physical and digital materials and artifacts) 
within and beyond the clinic $[5,10,20]$. Drawing on previous work on infrastructure" [17,18], we refer to this ongoing/dynamic work as "infrastructuring work" where multiple assembling entities have to work as a collective in order to make the often fragmented and broken healthcare services function for them.

Prior $\mathrm{HCl}$ studies have examined patients' work in a variety of settings, such as how patients engage in safety work where they try to detect and avoid medical errors during their hospital stays [19], information work to manage all verbal and written information received during cancer care [8], and interpretation work [1] to make sense of the self-measurements. In addition, a series of self-care work at home has been reported to sustain the care infrastructure, such as surveillance work while keeping track and monitoring their overall health [2], articulation work while handling medication $[3,4]$, machine work while setting and operating different monitoring devices [7,14], diagnosis work to identify possible health conditions online $[11,14]$, and emotional work to manage the impact of illness $[7,14]$.

While previous research focused on how people with chronic conditions experience and use technology for self-care to foster reflection, support treatment, and share experiences [16], our goal is to further understand health care infrastructures as a whole bringing attention to the spatial, material and relational practices people engage in when interacting with the larger, behind-the-scene healthcare service system through human and nonhuman arrangements. For instance, a recent Vox article points out that patients in the U.S. often encounter mysterious emergency visit bills that they often had no clue about, and urged them to submit their bills in order to puzzle out how medical payments work [9]. It raises important questions on how do people handle breakdowns in healthcare infrastructures and what can we do to design better interface system to connect patients with other associated entities of care infrastructures.

The fragmentation and inefficiency of healthcare infrastructure are not unique to the U.S. healthcare system. Across the globe, the same problem exists in many countries with distinctly different healthcare infrastructures (e.g., UK [13], India [21], Chile [15]). Patients and caregivers have no choice but to engage in "infrastructuring" work in order to align their needs, with the associated entities (human and non-human) of care infrastructure. However, the work patients do to construct their functioning health service systems often remains invisible, and very few resources are available to assist them Encountering healthcare infrastructure breakdowns alone feels like groping in the dark and leads to constant frustration, and emotional burden [6]. It is thus imperative to study the challenges patients and caregivers face (re)-configuring, connecting, navigating, constructing, adapting and sustaining the healthcare infrastructure including its spatial, material and relational practices, the types of work they do, and the strategies they develop to obtain the best quality care in interacting with professional healthcare service. 


\section{BACKGROUND}

From mundane, routine care such as common flu, childbirth, elderly health management, to lifethreaten conditions like cancers, encountering and interacting with healthcare service infrastructures is an everyday endeavor for many individuals worldwide. Yet interacting with healthcare service system can be extremely difficult and frustrating due to its fragmented nature and lack of information, trust and transparency. Considering the following examples of everyday care: new parents, pregnant women, older adults, and cancer patients. In these cases, to obtain satisfactory healthcare service, patients have to engage in a variety of work, such as transferring information and connecting service in between primary care, different specialties, and pharmacies; proactively figuring out various payment options and be prepared to handle unexpected bills and requests; monitoring the progress of care and service requests; and finding ways to compensate any delays and issues. In addition, with the use of digital systems, individuals also have to deal with and master various information resources and online systems to manage their interactions with the digital health infrastructures.

Meanwhile, the increasing popularity of digital health services are increasing the accessibility of information and services as never before, enabling greater agency and decision making, reshaping the boundaries between personal and professional care, often challenging the asymmetrical power relation between doctors and patients during consultations and treatment. Patients and caregivers are empowered with unique opportunities to participate and work towards functioning healthcare service system. Just as the case of collectively deciphering medical bills through digital media, citizens have new ways to access the information used to be inaccessible to them, and to find solutions to lower the costs of the healthcare service systems. Similarly, the recent emergence of online ask the doctors services also offers ways to interacting with health professionals and consult health issues that do not need an actual trip to care facilities [12]. These opportunities show the power of ICT systems and encourage us to brainstorm ways to address these issues through sociotechnical solutions (such as the case of deciphering medical bills through citizen participation) that can support and sustain healthcare infrastructures.

\section{WORKSHOP GOALS}

This workshop aims to bring together researchers, health practitioners, and patients to examine, discuss, and brainstorm ways to re-envision our healthcare service systems from the patients and caregivers' perspectives, and to unpack the types of work that they do to reconfigure, reconstruct and adapt the healthcare infrastructure. Specifically, we have the following goals:

- Identifying different types of work that individuals engage in making the professional service system functioning 
- Sharing experiences, resources and strategies patients and caregivers develop when encountering complex healthcare services, facing different regulations, and policies from healthcare services systems from an international perspective

- Reflecting on the role of materiality and spatiality and how they influence and transform both individual's and health professional's experiences with care infrastructures

- Leveraging the discussion from the workshop to brainstorm new thoughts into digital health system design that offers better connection than the traditional healthcare service infrastructure and to eliminate the burden individuals face in making the service work

- Providing inspiration from multidisciplinary, cross-geography and cultural, and trans-national perspectives into the design of new sociotechnical solutions to support and sustain care infrastructures

We believe our discussion could result in deeper understanding of the work needed in interacting and making health services work; providing insights on the material, spatial, and relational entities of care infrastructures as well as inform the design of digital systems that can support patients and caregivers' needs and lower the burden of their invisible work; offering new vision for the future of eHealth or Internet Health service; and reflecting, sharing and learning across different sociocultural and geographical healthcare systems and setting out research agendas for the future design of healthcare services with a global perspective.

\section{ORGANIZERS}

We are a team of internationally formed experts in studying patients' work and healthcare infrastructures. Our experiences span over multiple health conditions including women's health, elderly care, chronic illnesses, and a variety of offline, digital hybrid and online health service system across seven different countries. The combined expertise and experience of the organizers will provide valuable insights into different healthcare infrastructures, practices, and social cultural context, and provide transnational perspective into the design of future healthcare IT systems.

Yunan Chen is an associate professor in the Informatics Department, University of California Irvine. Yunan has done extensive research in $\mathrm{HCI}, \mathrm{CSCW}$, and health informatics. Her current work explores how individuals navigate the healthcare landscape using both online and offline resources in the U.S. Yunan has organized multiple $\mathrm{CHI}$ and $\mathrm{CSCW}$ workshops in the past.

Nervo Verdezoto is an assistant professor at the Department of Informatics, University of Leicester. His main research areas include $\mathrm{HCl}, \mathrm{CSCW}$ and Digital Health. His work has investigated the challenges and opportunities to support medication and self-monitoring practices of older adults. His current work explores how technologies shape pregnant women's experiences, clinical encounters and decision-making. Nervo has co-organized workshops for NordiCHI, ECSCW and CSCW. 
Xinning Gui is a PhD candidate in the Department of Informatics at University of California, Irvine. Her research lies in the intersection of health informatics, $\mathrm{HCl}$, and $\mathrm{CSCW}$. She adopts a socio-technical approach to examine patients and caregivers' interaction with the health care system in the contexts of individual service acquisition and public health.

Xiaojuan $\mathrm{Ma}$ is an assistant professor of Human-Computer Interaction $(\mathrm{HCl})$ at the Department of Computer Science and Engineering, Hong Kong University of Science and Technology. Her back ground is in $\mathrm{HCl}$ (CSCW, Ubicomp, etc.). She is particularly interested in data-driven human-engaged computing in the domain of health, education, and design. Xiaojuan has organized multiple workshops in various venues such as SIGGRAPH Asia in previous years. Claus Bossen is an associate professor in the Information Studies Department, Aarhus University. Claus has done extensive research in CSCW, Participatory Design and health informatics. His current work explores the work with data by patients, healthcare professionals and administrators to generate, transform and use of data for improvement of quality and efficiency. He has organized multiple CSCW and Participatory Design workshops in the past.

Naveen Bagalkot is a researcher and faculty member at the Srishti Institute of Art, Design and Technology, Bangalore, India. Naveen's current work explores co-design of healthcare technologies, particularly with and for underserved communities in the context of pregnancy care and care of chronic conditions in South India.

Valeria Herskovic is an associate professor at the Department of Computer Science, Pontificia Universidad Católica de Chile. Her research is the areas of $\mathrm{HCl}$ and health informatics, especially focused on older people and people with low digital skills in Chile. She also co-organizes the local women in computing conference, Chilewic.

Bernd Ploderer is a Lecturer in Human-Computer Interaction for Healthy Living at Queensland University of Technology (QUT). Through co-design with people with chronic conditions, caregivers, and clinicians, Bernd currently investigates how to improve the transition from hospital to home in Australia.

\section{REFERENCES}

[1] Tariq Andersen, Pernille Bjørn, Finn Kensing, and Jonas Moll. 2011. Designing for collaborative interpretation in telemonitoring: Re-introducing patients as diagnostic agents. International Journal of Medical Informatics 80, 8: e112-e126.

[2] Amid Ayobi, Paul Marshall, Anna L. Cox, and Yunan Chen. 2017. Quantifying the Body and Caring for the Mind: Self-Tracking in Multiple Sclerosis. Proc. of CHI 2017, ACM Press, 6889-6901.

[3] Sudeh Cheraghi-Sohi, Mark Jeffries, Fiona Stevenson, et al. 2015. The influence of personal communities on the self-management of medication taking: A wider exploration of medicine work. Chronic Illness 11, 2: 77-92. 
[4] Lea Dalgaard, Erik Grönvall, and Nervo Verdezoto. 2013. Accounting for Medication Particularities: Designing for Everyday Medication Management. Proceedings of the ICTs for improving Patients Rehabilitation Research Techniques, IEEE.

[5] Peter Danholt and Henriette Langstrup. 2012. Medication as Infrastructure: Decentring Self-care. Culture Unbound: Journal of Current Cultural Research 4, 3: 513-532.

[6] Xinning Gui, Yunan Chen, and Kathleen H. Pine. 2018. Navigating the Healthcare Service "Black Box": Individual Competence and Fragmented System. Proceedings of the ACM on HumanComputer Interaction. Vol 2, CSCW: Article 61.

[7] Elizabeth Kaziunas, Silvia Lindtner, Mark S. Ackerman, and Joyce M. Lee. 2018. Lived Data: Tinkering With Bodies, Code, and Care Work. Human-Computer Interaction 33, 1: 49-92.

[8] Predrag Klasnja, Andrea Civan Hartzler, Kent T Unruh, and Wanda Pratt. 2010. Blowing in the Wind: Unanchored Patient Information Work during Cancer Care. Proc. of CHI 2010, NIH Public Access, 193-202.

[9] Sarah Kliff. Hospital ER fees: They've been secret. We're uncovering them. Vox. Retrieved August 28, 2018 from https:/www.vox.com/2018/2/27/16936638/er-bills-emergency-room-hospital-feeshealth-care-costs

[10] Henriette Langstrup. 2013. Chronic care infrastructures and the home. Sociology of Health \& Illness 35, 7: 1008-1022.

[11] Deborah Lupton and Annemarie Jutel. 2015. 'It's like having a physician in your pocket!' A critical analysis of self-diagnosis smartphone apps. Social Science \& Medicine 133: 128-135.

[12] Xiaojuan Ma, Xinning Gui, Jiayue Fan, Mingqian Zhao, Yunan Chen, and Kai Zheng. 2018. Professional Medical Advice at your Fingertips: An empirical study of an online "Ask the Doctor" Platform. Proceedings of the ACM on Human-Computer Interaction. Vol 2, CSCW: Article 116.

[13] Nicola Mackintosh, Susanna Rance, Wendy Carter, and Jane Sandall. 2017. Working for patient safety: a qualitative study of women's help-seeking during acute perinatal events. BMC Pregnancy and Childbirth 17, 1: 232 .

[14] Nicola Mackintosh, Nervo Verdezoto, Sarah Q. Gong, and Michelle Hadjiconstantinou. 2018 Digitally mediated reproductive health across care boundaries. BSA Medical Sociology Group Annual Conference, British Sociological Association.

[15] Annick Manuel. 2002. The Chilean Health System: 20 Years of Reforms. Salud Pública de México 44, 1: 60-68.

[16] Francisco Nunes, Nervo Verdezoto, Geraldine Fitzpatrick, Morten Kyng, Erik Grönvall, and Cristiano Storni. 2015. Self-Care Technologies in $\mathrm{HCl}$ : Trends, Tensions, and Opportunities. ACM Transactions on Computer-Human Interaction 22, 6: 1-45. 
[17] Volkmar Pipek and Volker Wulf. 2009. Infrastructuring: Toward an Integrated Perspective on the Design and Use of Information Technology. Journal of the Association for Information Systems 10, 5: 447-473.

[18] Susan Leigh Star and Geoffrey C. Bowker. How to Infrastructure. In Handbook of New Media: Social Shaping and Consequences of ICTs. SAGE Publications, Ltd, 1 Oliver's Yard, 55 City Road London EC1Y 1SP, 151-162.

[19] Kenton T. Unruh and Wanda Pratt. 2007. Patients as actors: The patient's role in detecting, preventing, and recovering from medical errors. International Journal of Medical Informatics 76 : S236-S244.

[20] Kate Weiner and Catherine Will. 2018. Thinking with care infrastructures: people, devices and the home in home blood pressure monitoring. Sociology of Health \& Illness 40, 2: 270-282.

[21] Naveen Bagalkot, Nervo Verdezoto, Mitchelle Lewis, Paula Griffiths, Deirdre Harrington, Nicola Mackintosh, and Judith Angelitta Noronha.2018. Towards Enhancing Everyday Pregnancy Care: Reflections from Community Stakeholders in South India. Proc. of IndiaHCI 2018. 